\title{
Qualitative analysis of drinking water through the most probable number (MPN) method
}

\author{
Tasnia Ahmed, Sagar Baidya, Mrityunjoy Acharjee and Tasmina Rahman \\ Department of Microbiology, Stamford University Bangladesh, 51 Siddeswari Road, Dhaka 1217, \\ Bangladesh
}

Received 14 April 2013/Accepted 2 June 2013

\begin{abstract}
Water borne disease outbreaks associated with the drinking of unsafe water, containing pathogenic bacteria of fecal origin, is common in densely populated countries like Bangladesh. Present study was attempted to detect indicator bacteria from drinking water samples for the presumptive occurrence of fecal contaminations that are responsible for health associated problems. Therefore, a laboratory scale qualitative analysis through most probable number (MPN) method was employed. The indicator bacterium Escherichia coli were detected in 24 water samples out of 75 samples as revealed consequently by the presumptive, confirmed and completed tests of MPN method. Other Gram negative bacteria found in the samples included Alcaligenes faecalis, Pseudomonas spp., Klebsiella spp. and Proteus spp. Almost all of the identified bacteria showed resistance against commonly used antibiotics which is of significant health concern.
\end{abstract}

Key words: Drinking water; Most probable number (MPN); Microorganisms; Coliform; Fecal contamination

The scenario of water pollution imparted by the existence of microorganisms in drinking water is not unusual and the resultant disease endemics or epidemics are common vastly in the developing countries where sanitation, water purification and the practice of hygiene are ignored $(1,2)$. Dhaka, the capital of Bangladesh, is a over populated city having more than 2.5 million people with the increasing rate of about $5 \%$ each year (3). However, events of microbial contamination are frequent along this city resulting in various categories of enteric diseases heading from the consumption of water supplied by Dhaka Water Supply Authority (DWASA) without appropriate purification or processing $(1,2,4-8)$.

Mainly water supply in Dhaka metropolis is generated from the underground water (aquifer under 50 and 120 meters) sources without any treatment because of the lack of chance to microbial contamination from the outside environment $(1,2)$. Some water supplies may also serve water from surface water sources which are treated to remove contaminations before supplying in the distribution system. General people in Dhaka use water for drinking purposes either directly consuming or after some processing of the water by means of filtration or boiling $(1,2)$. The major health risk from

${ }^{\ddagger}$ Corresponding Author. Mailing address: Tasmina Rahman, Department of Microbiology, Stamford University Bangladesh, 51, Siddeswari Road, Dhaka 1217, Bangladesh; Email: tasmina_mb@yahoo.com. drinking water is caused by the presence or introduction of fecal coliforms in the drinking water supply which may come from the non-treated sewage systems sited nearly the water source or distribution system as well as overflow from them $(2,9-13)$. Coliform bacteria may account for public health associated diseases including diarrhea, dysentery, typhoid, salmonellosis, listeriosis, parasitic worm infestations, and viral infections are introduced from the diseased or carrier stage patients shedding such pathogens which by chance come into contact with the potable water systems (14-18).

The quality of drinking water can be tested by the processes determining the presence of different contaminating bacteria in the water sample which is usually time consuming as well as costly (7). Moreover, often the pathogens present are very low in number so that they could be missed during the testing procedures (19). A comparatively easy way to examine the water quality is to determine the presence of coliform bacteria serving as indicator, and hence the presence of such bacteria indicates the risk of pathogenic contamination from fecal origin $(20,21)$. The most common test for indicator organism is to determine the presence of Escherichia coli which only indicates the possibility of fecal contamination, not the actual presence of fecal pathogens as well as the occurrence of diseases (7).

However, for rapid detection of indicator organisms in drinking water, the most probable number (MPN) method, which is not that common in usage, might be considered 
$(22,23)$. It is actually a qualitative test rather than quantitative indicating only the presence of coliforms, not their numerical presentation. This test is carried out in three continuous stages: presumptive test, confirmed test and completed test through which the presence of indicator organism E. coli is detected and confirmed (24). The test is also known as multiple tube test which involves the use of multiple tubes to determine the presence of indicator organism using some key characteristics of them including - lactose fermentation producing gas, green metallic sheen on eosin methylene blue (EMB) agar and by Gram reaction observed in the mentioned three stages, respectively. Overall, with a shortfall of microbial enumeration, the MPN method of water quality detection is cost effective and rapid as well to determine the water quality. A previous work of Ahmed et al. (1) reported the MPN technique as a tool to evaluate water quality in a routine microbiological laboratory with public health significance. Along these lines, the present study employed MPN method to determine the microbiological quality of drinking water with an intension of further disseminating the technique in for broader application.

\section{MATERIALS AND METHODS}

Study area and sampling. The drinking water samples used by the residents of Dhaka metropolis were tested in the current study. Seventy five water samples were collected from separate household and commercial points which were used to consume after pre-treatments during the time period of January, 2013 to May, 2013. Samples were collected aseptically in sterile screw capped bottles maintaining in a thermal stabilizing box with a constant temperature of $25 \square \mathrm{C}$, transported to the laboratory within one hour, and immediately subjected to microbiological analysis $(1,2)$

MPN test protocol.

Presumptive test. Presumptive test involves the primary presumption for the presence of Gram negative coliform bacteria in the samples demonstrated by the appearance of gas in the lactose fermentation broth. For the presumptive test procedure 15 sets of test tubes containing lactose fermentation broth required for each sample under analysis. Each test tube contained $10 \mathrm{ml}$ of fermentation broth and inoculated with the water sample in a sequential order of $10 \mathrm{ml}$ in five of each $2 \mathrm{X}$ lactose fermentation broth, $1 \mathrm{ml}$ in five of each $1 \mathrm{x}$ lactose fermentation broth and lastly $0.1 \mathrm{ml}$ in five of each $10 \mathrm{ml} 1 \mathrm{X}$ lactose fermentation broth. All the test tubes were incorporated with Derhum tubes for detection of gas formation by Gram negative coliform bacteria. Test tubes were incubated with half circled screw caps at $37{ }^{\circ} \mathrm{C}$ for 48 hours. This procedure was followed for all of the 75 samples individually (1).

Confirmed test. Positive samples with the production of gas in the lactose fermentation broth were selected for the confirmed test procedures to detect the indicator bacteria of fecal origin Escherichia coli. EMB media was used to differentiate other Gram negative coliform bacteria from the Escherichia coli by the production of green metallic sheen in the media. The presence of green metallic sheen in EMB confirms the presence the indicator bacteria E. coli. One loopful sample from the positive test tubes was inoculated on EMB by streaking and incubated at $37{ }^{\circ} \mathrm{C}$ for 24 hours and then observed for the production of green metallic sheen (1).

Completed test. From the positive EMB plates showing green metallic sheen colonies of E. coli, the isolated colonies were inoculated into LFB $1 \mathrm{X}$ media containing Derhum tube to re-confirm the positive lactose fermentation. From the same colony, indicator organism (E. coli) was observed microscopically for their Gram reactions. This was the final stage of the MPN method where in the decision of water quality as potable or non-potable, could be made after confirmation and completion of the study (1). Finally, the standard biochemical tests were performed to confirm the identification of all the pathogenic isolates found in all 75 types of drinking water samples by the previously described methods $(24,25)$.

Enumeration of total viable bacteria and total fecal coliform. $0.1 \mathrm{~mL}$ of each sample suspension was spread onto nutrient agar (NA) for enumerating total viable bacteria (TVB). After incubation at $37 \square \mathrm{C}$ for 24 hours, plates were examined. For estimating the total fecal coliform bacteria, $100 \mathrm{ml}$ of water sample was passed through the membrane filter which was then put over MFC medium and incubated at $44.5^{\circ} \mathrm{C}$ for 48 hours.

Antibiotic susceptibility test of the identified bacteria. The pathogenic isolates were examined for antibiotic susceptibility traits (either drug resistant or sensitive) by disc diffusion assay on Mueller-Hinton agar (Difco, Detroit, MI) against commonly used antibiotics following the standard protocol $(17,26,27)$. Antibiotics used in the study included trimethoprime/sulfamethoxazole $(25 \mu \mathrm{g})$, erythromycin $(15 \mu \mathrm{g})$, amoxicillin $(30 \mu \mathrm{g})$, ceftriaxone $(30 \mu \mathrm{g})$, ciprofloxacin $(5 \mu \mathrm{g})$, streptomycin $(10 \mu \mathrm{g})$, ampicillin $(10 \mu \mathrm{g})$, tetracycline $(30 \mu \mathrm{g})$, chloramphenicol (30 $\mu \mathrm{g})$, cefixime $(5 \mu \mathrm{g})$, polymyxin B (300 units), kanamycin $(30 \mu \mathrm{g})$, vancomycin $(30 \mu \mathrm{g})$, gentamycin $(10 \mu \mathrm{g})$, nalidixic acid $(30 \mu \mathrm{g})$, azythromycin $(15 \mu \mathrm{g})$ and penicillin $\mathrm{G}(10 \mu \mathrm{g})$.

\section{RESULTS AND DISCUSSION}

Poor water quality, sanitation and hygiene account for about 2 million deaths a year world-wide $(3.1 \%$ of all deaths and $3.7 \%$ of daily), mainly through infectious diarrhea $(4,17)$. Such diseases are more common in developing countries like Bangladesh due to poor quality of drinking water $(1,2,8,17)$. Thorough checking of drinking water quality even after application of some treatment such as boiling, filtration etc. is therefore necessary for minimizing the public health risk and initiating proper maintenance approaches. Present experiment was conducted for the determination of the processed drinking water quality to be either potable or non-potable on the basis of the presence of indicator bacteria which indicates the chance of fecal contamination as well as the health associated risks. This identification procedure was done by applying the MPN method which is rather cheap and less time consuming method in the context of developing countries. Another facet of our study was the detection of drug resistance among the pathogenic isolates which were also found along with $E$. coli.

Bacteriological quality of the drinking water samples tested. All of the waters samples used in the current study were heavily contaminated with lactose fermentation positive bacteria which were determined by the formation of gas in the Derhum tube after 48 hours of incubation at $37^{\circ} \mathrm{C}$ (Table 1). Sample no. 01, 03, 19, 28, 33, 34, 46, 50, $57,62,65,67,69,70,73$ and 74 showed maximum counts of positive results for each of the 15 test tubes by observing the formation of gas resulting $\geq 2400 \mathrm{MPN} / 100$ $\mathrm{ml}$ of sample. Sample no. 29 and 32 showed lowest count as $2 \mathrm{MPN} / 100 \mathrm{~mL}$ of sample.

21 samples (sample no. 1, 11, 13, 17, 18, 21, 24, 28, 36, $46,47,48,51,52,53,54,55,56,57,58$ \& 59) exhibited green metallic sheen on EMB media indicating the presence of fecal coliform i.e. E. coli making the water sample non-potable (Table 2). In the completed test, all 21 E. coli isolates were further confirmed by their Gram reaction as Gram negative, short rod cells visualized under bright field microscope and by observing the formation of gas in lactose fermentation broth (Table 3). The presence of the indicator bacteria indicated the possible occurrence of fecal contamination. 
TABLE 1. Results of presumptive test

\begin{tabular}{|c|c|c|c|c|c|c|}
\hline $\begin{array}{c}\text { Sample } \\
\text { No. }\end{array}$ & $\begin{array}{c}5 \text { of } 10 \mathrm{ml} \\
\text { each }\end{array}$ & $\begin{array}{c}5 \text { of } 1 \mathrm{ml} \\
\text { each }\end{array}$ & $\begin{array}{c}5 \text { of } 0.1 \mathrm{ml} \\
\text { each }\end{array}$ & $\begin{array}{l}\text { MPN/ } \\
100 \mathrm{ml}\end{array}$ & $\begin{array}{l}\text { Lower limit of } \\
95 \% \text { Confidence }\end{array}$ & $\begin{array}{l}\text { Upper limit of } \\
95 \% \text { confidence }\end{array}$ \\
\hline 1 & 5 & 5 & 5 & $\geq 2400$ & 700 & 0 \\
\hline 2 & 5 & 5 & 4 & 1600 & 400 & 4600 \\
\hline 3 & 5 & 5 & 5 & $\geq 2400$ & 700 & 0 \\
\hline 4 & 5 & 5 & 3 & 920 & 220 & 2600 \\
\hline 5 & 5 & 3 & 2 & 140 & 52 & 400 \\
\hline 6 & 5 & 1 & 1 & 46 & 14 & 120 \\
\hline 7 & 5 & 2 & 1 & 70 & 22 & 170 \\
\hline 8 & 5 & 2 & 1 & 70 & 22 & 170 \\
\hline 9 & 5 & 5 & 1 & 350 & 100 & 710 \\
\hline 10 & 4 & 4 & 1 & 21 & 7 & 42 \\
\hline 11 & 5 & 4 & 0 & 130 & 36 & 400 \\
\hline 12 & 4 & 0 & 0 & 13 & 4 & 35 \\
\hline 13 & 4 & 1 & 2 & 26 & 7 & 60 \\
\hline 14 & 5 & 5 & 4 & 1600 & 400 & 4600 \\
\hline 15 & 5 & 4 & 2 & 220 & 70 & 440 \\
\hline 16 & 5 & 5 & 4 & 1600 & 400 & 4600 \\
\hline 17 & 5 & 4 & 1 & 170 & 58 & 100 \\
\hline 18 & 3 & 1 & 0 & 11 & 5 & 35 \\
\hline 19 & 5 & 5 & 5 & $\geq 2400$ & 700 & 0 \\
\hline 20 & 5 & 5 & 3 & 920 & 220 & 2600 \\
\hline 21 & 5 & 1 & 1 & 46 & 14 & 120 \\
\hline 22 & 4 & 0 & 0 & 13 & 4 & 35 \\
\hline 23 & 3 & 0 & 0 & 8 & 2 & 22 \\
\hline 24 & 2 & 0 & 0 & 5 & $<0.5$ & 13 \\
\hline 25 & 5 & 2 & 2 & 220 & 70 & 440 \\
\hline 26 & 1 & 0 & 0 & 2 & 0.3 & 10 \\
\hline 27 & 5 & 5 & 2 & 540 & 150 & 1700 \\
\hline 28 & 5 & 5 & 5 & $\geq 2400$ & 700 & 0 \\
\hline 29 & 1 & 0 & 0 & 2 & 0.3 & 10 \\
\hline 30 & 4 & 4 & 0 & 34 & 11 & 90 \\
\hline 31 & 2 & 0 & 1 & 7 & 1 & 17 \\
\hline 32 & 1 & 0 & 0 & 2 & 0.3 & 10 \\
\hline 33 & 5 & 5 & 5 & $\geq 2400$ & 700 & 0 \\
\hline 34 & 5 & 5 & 5 & $\geq 2400$ & 700 & 0 \\
\hline 35 & 4 & 3 & 0 & 33 & 9 & 70 \\
\hline 36 & 2 & 0 & 0 & 5 & $<0.5$ & 13 \\
\hline 37 & 5 & 4 & 0 & 130 & 36 & 400 \\
\hline 38 & 3 & 0 & 0 & 8 & 2 & 22 \\
\hline 39 & 2 & 0 & 0 & 5 & $<0.5$ & 13 \\
\hline 40 & 3 & 0 & 0 & 8 & 2 & 22 \\
\hline 41 & 5 & 3 & 0 & 79 & 22 & 220 \\
\hline 42 & 1 & 1 & 0 & 4 & 0.7 & 12 \\
\hline 43 & 1 & 1 & 0 & 4 & 0.7 & 12 \\
\hline 44 & 4 & 1 & 0 & 17 & 6 & 40 \\
\hline 45 & 2 & 0 & 0 & 5 & $<0.5$ & 13 \\
\hline 46 & 5 & 5 & 5 & $\geq 2400$ & 700 & 0 \\
\hline 47 & 5 & 4 & 1 & 170 & 58 & 100 \\
\hline 48 & 5 & 1 & 0 & 33 & 10 & 100 \\
\hline 49 & 5 & 5 & 2 & 540 & 150 & 1700 \\
\hline 50 & 5 & 5 & 5 & $\geq 2400$ & 700 & 0 \\
\hline 51 & 5 & 3 & 1 & 110 & 34 & 250 \\
\hline 52 & 5 & 3 & 1 & 110 & 34 & 250 \\
\hline 53 & 4 & 1 & 0 & 17 & 6 & 40 \\
\hline 54 & 5 & 4 & 0 & 130 & 36 & 400 \\
\hline 55 & 5 & 1 & 0 & 33 & 10 & 100 \\
\hline 56 & 5 & 1 & 1 & 46 & 14 & 120 \\
\hline 57 & 5 & 5 & 5 & $\geq 2400$ & 700 & 0 \\
\hline 58 & 5 & 0 & 0 & 23 & 7 & 70 \\
\hline 59 & 5 & 0 & 0 & 23 & 7 & 70 \\
\hline 60 & 5 & 0 & 0 & 23 & 7 & 70 \\
\hline
\end{tabular}


TABLE 1. Continued ...

\begin{tabular}{ccccccc}
\hline $\begin{array}{c}\text { Sample } \\
\text { No. }\end{array}$ & $\begin{array}{c}5 \text { of } 10 \mathrm{ml} \\
\text { each }\end{array}$ & $\begin{array}{c}5 \text { of } 1 \mathrm{ml} \\
\text { each }\end{array}$ & $\begin{array}{c}5 \text { of } 0.1 \mathrm{ml} \\
\text { each }\end{array}$ & $\begin{array}{c}\text { MPN/ } \\
100 \mathrm{ml}\end{array}$ & $\begin{array}{c}\text { Lower limit of } \\
95 \% \text { Confidence }\end{array}$ & $\begin{array}{c}\text { Upper limit of } \\
95 \% \text { confidence }\end{array}$ \\
\hline 61 & 5 & 5 & 3 & 920 & 220 & 2600 \\
62 & 5 & 5 & 5 & $\geq 2400$ & 700 & 0 \\
63 & 5 & 0 & 0 & 23 & 7 & 70 \\
64 & 4 & 2 & 0 & 22 & 7 & 50 \\
65 & 5 & 5 & 5 & $\geq 2400$ & 700 & 0 \\
66 & 5 & 5 & 0 & 240 & 70 & 710 \\
67 & 5 & 5 & 5 & $\geq 2400$ & 700 & 0 \\
68 & 5 & 5 & 2 & 540 & 150 & 0 \\
69 & 5 & 5 & 5 & $\geq 2400$ & 700 & 0 \\
70 & 5 & 5 & 5 & $\geq 2400$ & 700 & 170 \\
71 & 5 & 2 & 1 & 70 & 22 & 70 \\
72 & 5 & 0 & 0 & 23 & 7 & 0 \\
73 & 5 & 5 & 5 & $\geq 2400$ & 700 & 0 \\
74 & 5 & 5 & 5 & $\geq 2400$ & 700 & 100 \\
75 & 5 & 1 & 0 & 33 & 10 & \\
\hline
\end{tabular}

All the experiments have been done three times and the results were reproducible. One representative data have been shown.

TABLE 2. Results of confirmed test

\begin{tabular}{|c|c|c|c|c|c|c|c|}
\hline $\begin{array}{c}\text { Sample } \\
\text { No. }\end{array}$ & $\begin{array}{l}\text { Growth } \\
\text { on EMB }\end{array}$ & $\begin{array}{c}\text { Production of } \\
\text { greenish metallic } \\
\text { Sheen }\end{array}$ & Result & Sample & $\begin{array}{l}\text { Growth } \\
\text { on EMB }\end{array}$ & $\begin{array}{c}\text { Production of } \\
\text { greenish metallic } \\
\text { Sheen }\end{array}$ & Result \\
\hline 1 & + & + & Non potable & 39 & + & - & Potable \\
\hline 2 & + & - & Potable & 40 & + & - & Potable \\
\hline 3 & + & - & Potable & 41 & + & - & Potable \\
\hline 4 & + & - & Potable & 42 & + & - & Potable \\
\hline 5 & + & - & Potable & 43 & + & - & Potable \\
\hline 6 & + & - & Potable & 44 & + & - & Potable \\
\hline 7 & + & - & Potable & 45 & + & - & Potable \\
\hline 8 & + & - & Potable & 46 & + & + & Non potable \\
\hline 9 & + & - & Potable & 47 & + & + & Non potable \\
\hline 10 & + & - & Potable & 48 & + & + & Non potable \\
\hline 11 & + & + & Non potable & 49 & + & - & Potable \\
\hline 12 & + & - & Potable & 50 & + & - & Potable \\
\hline 13 & + & + & Non potable & 51 & + & + & Non potable \\
\hline 14 & + & - & Potable & 52 & + & + & Non potable \\
\hline 15 & + & - & Potable & 53 & + & + & Non potable \\
\hline 16 & + & - & Potable & 54 & + & + & Non potable \\
\hline 17 & + & + & Non potable & 55 & + & + & Non potable \\
\hline 18 & + & + & Non potable & 56 & + & + & Non potable \\
\hline 19 & + & - & Potable & 57 & + & + & Non potable \\
\hline 20 & + & - & Potable & 58 & + & + & Non potable \\
\hline 21 & + & + & Non potable & 59 & + & + & Non potable \\
\hline 22 & + & - & Potable & 60 & + & - & Potable \\
\hline 23 & + & - & Potable & 61 & + & - & Potable \\
\hline 24 & + & + & Non potable & 62 & + & - & Potable \\
\hline 25 & + & - & Potable & 63 & + & - & Potable \\
\hline 26 & + & - & Potable & 64 & + & - & Potable \\
\hline 27 & + & - & Potable & 65 & + & - & Potable \\
\hline 28 & + & + & Non potable & 66 & + & - & Potable \\
\hline 29 & + & - & Potable & 67 & + & - & Potable \\
\hline 30 & + & - & Potable & 68 & + & - & Potable \\
\hline 31 & + & - & Potable & 69 & + & - & Potable \\
\hline 32 & + & - & Potable & 70 & + & - & Potable \\
\hline 33 & + & - & Potable & 71 & + & - & Potable \\
\hline 34 & + & - & Potable & 72 & + & - & Potable \\
\hline 35 & + & - & Potable & 73 & + & - & Potable \\
\hline 36 & + & + & Non potable & 74 & + & - & Potable \\
\hline 37 & + & - & Potable & 75 & + & - & Potable \\
\hline 38 & + & - & Potable & & & & \\
\hline
\end{tabular}

All the experiments have been done three times and the results were reproducible. One representative data have been shown. 
TABLE 3. Completed test results (confirmation of E. coli)

\begin{tabular}{cccccccc}
\hline Sample No. & LB +/- & Gram reaction & Result & Sample & LB +/- & Gram reaction & Result \\
\hline 01 & + & Gram (-), short rod & E. coli confirmed & 48 & + & Gram (-), short rod & E. coli confirmed \\
11 & + & Gram (-), short rod & E. coli confirmed & 51 & + & Gram (-), short rod & E. coli confirmed \\
13 & + & Gram (-), short rod & E. coli confirmed & 52 & + & Gram (-), short rod & E. coli confirmed \\
17 & + & Gram (-), short rod & E. coli confirmed & 53 & + & Gram (-), short rod & E. coli confirmed \\
18 & + & Gram (-), short rod & E. coli confirmed & 54 & + & Gram (-), short rod & E. coli confirmed \\
21 & + & Gram (-), short rod & E. coli confirmed & 55 & + & Gram (-), short rod & E. coli confirmed \\
24 & + & Gram (-), short rod & E. coli confirmed & 56 & + & Gram (-), short rod & E. coli confirmed \\
28 & + & Gram (-), short rod & E. coli confirmed & 57 & + & Gram (-), short rod & E. coli confirmed \\
36 & + & Gram (-), short rod & E. coli confirmed & 58 & + & Gram (-), short rod & E. coli confirmed \\
46 & + & Gram (-), short rod & E. coli confirmed & 59 & + & Gram (-), short rod & E. coli confirmed \\
47 & + & Gram (-), short rod & E. coli confirmed & & & & \\
\hline
\end{tabular}

LB $=$ Lactose broth

All the experiments have been done three times and the results were reproducible. One representative data have been shown.

TABLE 4. Biochemical tests of the isolates other than E. coli found on EMB agar

\begin{tabular}{|c|c|c|c|c|c|c|c|c|c|c|c|}
\hline \multirow[b]{2}{*}{$\begin{array}{c}\text { Assumed } \\
\text { microorganisms }\end{array}$} & \multicolumn{4}{|c|}{ TSI } & \multirow[b]{2}{*}{ Motility } & \multirow[b]{2}{*}{$\begin{array}{c}\text { Indole } \\
\text { Production }\end{array}$} & \multirow[b]{2}{*}{ MR } & \multirow[b]{2}{*}{ VP } & \multirow[b]{2}{*}{$\begin{array}{c}\text { Citrate } \\
\text { utilization }\end{array}$} & \multirow[b]{2}{*}{$\begin{array}{c}\text { Catalase } \\
\text { test }\end{array}$} & \multirow[b]{2}{*}{$\begin{array}{c}\text { Oxidase } \\
\text { test }\end{array}$} \\
\hline & $\frac{\vec{\Xi}}{\tilde{n}}$ & 志 & $\mathbb{\mathscr { J }}$ & $\underbrace{\infty}$ & & & & & & & \\
\hline Klebsiella spp. & $\mathrm{Y}$ & $\mathrm{Y}$ & + & - & + & - & - & + & + & $+\mathrm{ve}$ & -ve \\
\hline Alcaligenes faecalis & $\mathrm{Y}$ & $\mathrm{Y}$ & + & - & + & - & - & - & + & $+\mathrm{ve}$ & $+\mathrm{ve}$ \\
\hline Pseudomonas spp. & $\mathrm{R}$ & $\mathrm{R}$ & - & - & - & - & - & - & + & $+\mathrm{ve}$ & $+\mathrm{ve}$ \\
\hline Aeromonas spp. & $\mathrm{R}$ & $\mathrm{Y}$ & + & - & + & - & - & + & + & $+\mathrm{ve}$ & $+\mathrm{ve}$ \\
\hline Proteus spp. & $\mathrm{Y}$ & $\mathrm{Y}$ & + & + & + & - & - & + & + & $+\mathrm{ve}$ & -ve \\
\hline Vibrio spp. & $\mathrm{R}$ & A & - & - & + & - & - & - & + & $+\mathrm{ve}$ & $+\mathrm{ve}$ \\
\hline
\end{tabular}

TSI = Triple sugar iron test; $\mathrm{Y}=$ Yellow (Acid); $\mathrm{R}=$ Red (Alkaline); MR = Methyl red; VP = Voges-Proskauer

To demonstrate the full profile of microbial contamination of the water samples, other contaminating bacteria besides Escherichia coli were identified and confirmed by biochemical determination (Table 4). Proteus spp., Alcaligenes spp., Klebsiella spp., Pseudomonas spp., Alcaligenes faecalis, Aeromonas spp. and Vibrio spp. were identified after conducting the biochemical tests (Table 4). Klebsiella spp. and Aeromonas spp. were found to be the prominent microorganisms in most of the samples. Vibrio spp. (only in sample 24) and Aeromonas spp. (samples no. 9, 17) was found in few samples. Pseudomonas spp. was detected in four samples (sample no. 7, 22, 29 and 66). Processing drinking water by more sophisticated ways and also by detecting the ways of contamination, the degree of such health problems might be lessened.

Total aerobic plate count was performed to understand the overall microbiological condition of the samples and quantification of total fecal coliform was conducted to determine the associated risk with other possible pathogens. All samples contained total aerobic bacterial load ranging from $1 \times 10^{4}$ to $3 \times 10^{6} \mathrm{cfu} / \mathrm{ml}$ (Table 5). Although fecal coliform was detected only in one sample (sample no. 54), such findings could verify the MPN results.

Proliferation of drug-resistant bacteria in drinking water. To project the clinical significance of the bacterial isolates found in this study, a further study of the contaminants' drug-resistance trait was conducted. Identified bacterial isolates were experimented to determine the antibiotic susceptibility against the commonly therapeutically used antibiotics. Alcaligenes faecalis showed susceptibility towards all of the commonly used antibiotics experimented during this study (Table 6). Vibrio spp. showed resistance against most of the commonly consumed antibiotics, than the other isolates. As water can be accessed easily by a variety of ways to different environmental conditions, the resistance gene from the drug resistant bacteria to other wild type (sensitive) bacterial isolates, expanding the resistant behavior to a broader range of species of bacteria $(1,2$, 17).

In our study, higher resistance was observed against azythromycin $(15 \mu \mathrm{g})$, cefixime $(5 \mu \mathrm{g})$, ampicillin $(10 \mu \mathrm{g})$, penicillin $\mathrm{G}(10 \mu \mathrm{g})$, and nalidixic acid $(30 \mu \mathrm{g})$, while some were intermediate resistant against ceftriaxon $(30 \mu \mathrm{g})$, streptomycin $(10 \mu \mathrm{g})$, and kanamycine $(30 \mu \mathrm{g})$. Resistance 
TABLE 5. Total viable bacteria and total fecal coliform count

\begin{tabular}{|c|c|c|c|c|c|}
\hline Sample & $\begin{array}{c}\text { TVB count } \\
\mathrm{cfu} / \mathrm{ml}\end{array}$ & $\begin{array}{l}\text { TFC count } \\
\mathrm{cfu} / 100 \mathrm{ml}\end{array}$ & Sample & $\begin{array}{l}\text { TVB count } \\
\text { cfu/ml }\end{array}$ & $\begin{array}{l}\text { TFC count } \\
\text { cfu/ } 100 \mathrm{ml}\end{array}$ \\
\hline 1 & $2.6 \times 10^{5}$ & - & 39 & $8.0 \times 10^{4}$ & - \\
\hline 2 & $2.3 \times 10^{5}$ & - & 40 & $3.0 \times 10^{5}$ & - \\
\hline 3 & $1.8 \times 10^{6}$ & - & 41 & $7.8 \times 10^{5}$ & - \\
\hline 4 & $1.7 \times 10^{5}$ & - & 42 & $1.2 \times 10^{5}$ & - \\
\hline 5 & $1.1 \times 10^{5}$ & - & 43 & $1.1 \times 10^{5}$ & - \\
\hline 6 & $2.9 \times 10^{6}$ & - & 44 & $1.6 \times 10^{5}$ & - \\
\hline 7 & $1.6 \times 10^{6}$ & - & 45 & $4.0 \times 10^{4}$ & - \\
\hline 8 & $3.0 \times 10^{5}$ & - & 46 & $2.8 \times 10^{5}$ & - \\
\hline 9 & $1.5 \times 10^{5}$ & - & 47 & $1.4 \times 10^{5}$ & - \\
\hline 10 & $1.2 \times 10^{5}$ & - & 48 & $7.8 \times 10^{5}$ & - \\
\hline 11 & $2.1 \times 10^{6}$ & - & 49 & $1.4 \times 10^{5}$ & - \\
\hline 12 & $2.2 \times 10^{6}$ & - & 50 & $2.8 \times 10^{5}$ & - \\
\hline 13 & $2.6 \times 10^{5}$ & - & 51 & $7.8 \times 10^{5}$ & - \\
\hline 14 & $1.9 \times 10^{6}$ & - & 52 & $3.0 \times 10^{6}$ & - \\
\hline 15 & $1.4 \times 10^{6}$ & - & 53 & $2.7 \times 10^{6}$ & - \\
\hline 16 & $1.0 \times 10^{5}$ & - & 54 & $1.2 \times 10^{6}$ & - \\
\hline 17 & $2.3 \times 10^{5}$ & - & 55 & $1.1 \times 10^{6}$ & - \\
\hline 18 & $7.2 \times 10^{5}$ & - & 56 & $1.1 \times 10^{6}$ & - \\
\hline 19 & $8.3 \times 10^{5}$ & - & 57 & $8.8 \times 10^{5}$ & - \\
\hline 20 & $9.2 \times 10^{5}$ & - & 58 & $2.3 \times 10^{6}$ & - \\
\hline 21 & $9.9 \times 10^{5}$ & - & 59 & $1.3 \times 10^{6}$ & - \\
\hline 22 & $8.6 \times 10^{5}$ & - & 60 & $9.8 \times 10^{5}$ & - \\
\hline 23 & $2.6 \times 10^{6}$ & - & 61 & $1.8 \times 10^{6}$ & - \\
\hline 24 & $1.8 \times 10^{6}$ & - & 62 & $4.2 \times 10^{5}$ & - \\
\hline 25 & $1.5 \times 10^{6}$ & - & 63 & $3.0 \times 10^{5}$ & - \\
\hline 26 & $2.9 \times 10^{5}$ & - & 64 & $6.2 \times 10^{4}$ & - \\
\hline 27 & $6.0 \times 10^{4}$ & - & 65 & $2.9 \times 10^{6}$ & - \\
\hline 28 & $2.9 \times 10^{5}$ & - & 66 & $1.1 \times 10^{6}$ & - \\
\hline 29 & $2.8 \times 10^{5}$ & - & 67 & $1.3 \times 10^{6}$ & - \\
\hline 30 & $9.8 \times 10^{4}$ & - & 68 & $6.0 \times 10^{5}$ & - \\
\hline 31 & $1.0 \times 10^{4}$ & - & 69 & $2.8 \times 10^{5}$ & - \\
\hline 32 & $2.9 \times 10^{5}$ & - & 70 & $1.6 \times 10^{6}$ & - \\
\hline 33 & $1.4 \times 10^{5}$ & - & 71 & $7.6 \times 10^{5}$ & - \\
\hline 34 & $7.2 \times 10^{5}$ & - & 72 & $2.7 \times 10^{6}$ & - \\
\hline 35 & $2.6 \times 10^{5}$ & - & 73 & $1.4 \times 10^{5}$ & - \\
\hline 36 & $4.6 \times 105$ & - & 74 & $1.7 \times 10^{6}$ & - \\
\hline 37 & $2.2 \times 10^{5}$ & - & 75 & $5.8 \times 10^{5}$ & - \\
\hline 38 & $2.0 \times 10^{4}$ & - & & & \\
\hline
\end{tabular}

$\mathrm{TVB}=$ total viable bacteria; $\mathrm{TFC}=$ total fecal coliform

All the experiments have been done three times and the results were reproducible. One representative data have been shown.

gene might be evolved due to point mutation, genetic disorders, mechanistic factors or by epidemiological factors (28-30).

Finally, our study reported the non-potability of some processed drinking water samples (21) by observing the indicator bacteria $E$. coli which also indicated the possible presence of other pathogenic bacteria. Several factors such as environmental contamination, inadequate processing and improper handling etc. might be responsible for contamination of drinking water $(1,2)$. Besides, the presence drug resistance traits in the identified isolates might be responsible for the difficulties in eradicating the associated diseases commonly treated with those antibiotics $(1,31-33)$.

\section{CONCLUSIONS}

Being a densely populated developing country, Bangladesh has long been suffering from the disease complications of her subjects due to poor, unhygienic management of environment and water bodies. Poor economy, lack of education and training, unwillingness to employ the corrective measures, reluctance towards legislations set by the policies for water and environment, and finally the extraordinary burden of wastes saturated with pathogenic microorganisms could account for serious public health damage. Creating a sustainable environment with sound dissemination of water supply thus cruise the current interest of researchers involved in the application of scientific common sense. In our current research, we detected indicators in the drinking water samples and also identified other Gram negative bacteria present in the samples with their antibiotic susceptibly test towards commonly consumed antibiotics used for treatment mostly to portray a complete microbiological profile of the drinking water. By ensuring a complete regulation of microbiological quality of water in broader perspective, the maintenance and fortification of aquatic environments 
TABLE 6. Antibacterial susceptibility of the pathogenic isolates

\begin{tabular}{|c|c|c|c|c|c|c|c|c|}
\hline Antibiotic & $\begin{array}{l}\overrightarrow{0} \\
\stackrel{0}{0} \\
0 \\
0 \\
.00 \\
0\end{array}$ & 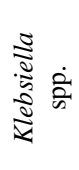 & $\begin{array}{l}\dot{2} \\
0 \\
0 \\
0 \\
0 \\
0\end{array}$ & 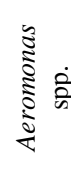 & 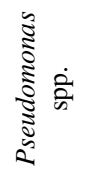 & 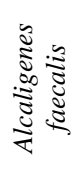 & $\begin{array}{l}\tilde{8} \\
\dot{0}\end{array}$ & 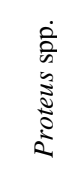 \\
\hline Polymyxin B & 300 units & $\mathrm{S}$ & $\mathrm{ND}$ & $\mathrm{S}$ & ND & ND & $\mathrm{S}$ & ND \\
\hline Kanamycin & $30 \mu \mathrm{g}$ & ND & I & ND & ND & ND & ND & $\mathrm{R}$ \\
\hline Vancomycin & $30 \mu \mathrm{g}$ & ND & $\mathrm{R}$ & ND & $\mathrm{S}$ & $\mathrm{S}$ & ND & ND \\
\hline Gentamicin & $10 \mu \mathrm{g}$ & ND & ND & ND & ND & ND & ND & $\mathrm{S}$ \\
\hline Nalidixic acid & $30 \mu \mathrm{g}$ & ND & $\mathrm{R}$ & ND & ND & ND & ND & ND \\
\hline Azythromycin & $15 \mu \mathrm{g}$ & ND & $\mathrm{R}$ & ND & ND & ND & ND & $\mathrm{R}$ \\
\hline Penicillin G & $10 \mu \mathrm{g}$ & ND & $\mathrm{R}$ & ND & ND & ND & ND & ND \\
\hline Trimethoprime/sulfamethoxazole & $25 \mu \mathrm{g}$ & $\mathrm{S}$ & ND & $\mathrm{R}$ & ND & ND & S & ND \\
\hline Erythromycin & $15 \mu \mathrm{g}$ & S & ND & $\mathrm{R}$ & ND & S & $\mathrm{R}$ & $\mathrm{R}$ \\
\hline Amoxicillin & $30 \mu \mathrm{g}$ & $\mathrm{R}$ & $\mathrm{R}$ & S & ND & S & S & $\mathrm{R}$ \\
\hline Ceftriaxone & $30 \mu \mathrm{g}$ & $\mathrm{R}$ & I & I & ND & S & S & ND \\
\hline Ciprofloxacin & $5 \mu \mathrm{g}$ & S & $\mathrm{R}$ & S & $\mathrm{S}$ & S & S & $\mathrm{S}$ \\
\hline Streptomycin & $10 \mu \mathrm{g}$ & $\mathrm{R}$ & I & S & ND & ND & I & ND \\
\hline Ampicillin & $10 \mu \mathrm{g}$ & $\mathrm{R}$ & $\mathrm{R}$ & S & $\mathrm{S}$ & ND & $\mathrm{R}$ & $\mathrm{S}$ \\
\hline Tetracyclin & $30 \mu \mathrm{g}$ & S & $\mathrm{R}$ & $\mathrm{S}$ & $\mathrm{S}$ & ND & S & $\mathrm{S}$ \\
\hline Chloramphenicol & $30 \mu \mathrm{g}$ & $\mathrm{S}$ & $\mathrm{S}$ & $\mathrm{R}$ & ND & ND & $\mathrm{S}$ & $\mathrm{S}$ \\
\hline Cefixime & $5 \mu \mathrm{g}$ & $\mathrm{R}$ & ND & $\mathrm{R}$ & ND & ND & $\mathrm{S}$ & $\mathrm{R}$ \\
\hline
\end{tabular}

$\mathrm{R}=$ Resistant $; \mathrm{S}=$ Sensitive; $\mathrm{I}=$ Intermediate $\mathrm{ND}$ = Not done

might also be maintained to a significant extent.

\section{ACKNOWLEDGEMENTS}

The work was financially supported by the department of Microbiology, Stamford University Bangladesh.

\section{REFERENCES}

1. Ahmed, T., et al. 2013. Microbiological study of drinking water: Qualitative and quantitative approach. Asian J. Microbiol. Biotech. Env. Sc. 15 (4): 647-654.

2. Acharjee, M., F. Jahan, F. Rahman, nd R. Noor. 2013. Bacterial proliferation in municipal water supplied in Mirpur locality of Dhaka City, Bangladesh. Clean - Soil, Air, Water 41: 1-8.

3. Haq, A. K. 2006. Water management in Dhaka. Water Resource Development 22 (2): 291-311

4. Mead, A. M., G. Helm, P. Callan, and R. M. Atlas. 1999. A prospective study of drinking water quality and gastrointestinal diseases. New Eng. J. Med. 245 (9): 224-248.

5. Kamal, M. M. A., M. Hansen and A. B. M. Badruzzaman. 1999. Assessment of pollution of the river Buriganga, using a water quality model. Water Sci. Tech. 40 (2): 129-136.

6. Subramania, B. 2004. Water quality in South Asia. Asian J. Water Environ. Pol. 1 (1-2): 41-55.

7. Mahbub, K. R., A. Nahar, M. M. Ahmed, and A. Chakraborty. 2011. Quality Analysis of Dhaka WASA drinking water: Detection and biochemical characterization of the isolates. J. Environ. Sci. Natural Res. 4 (2): 41-49.

8. Acharjee, M., et al. 2012. Microbiological study on supply water and treated water in Dhaka city. Stam. J. Microbial. 1 (1): 42-45.

9. Parveen, S., M. S. U. Ahmed, and N. Tania. 2008. Microbial contamination of water in around Dhaka city. Bang. J. Sci. Industr. Res. 43 (2): 273-276.

10. DiPaola, D. S. 1998. Biological and chemical renovation of waste water with a soil infilterator low- pressure distribution system, Virginia Tech. University Press, Virginia, EPA.

11. McLellan, S. L. 2004. Genetic diversity of Escherichia coli isolated from urban rivers and beach water. Appl. Environ. Microbiol. 70: 4658-4665.
12. Ahmed, W., R. Neller, and M. Katouli. 2005. Hpst- species specific metabolic fingerprint database for enetrococci and Escherichia coli and it application to identify sources of fecal contamination in surface waters. Appl. Environ. Microbiol. 71: 4461-4468.

13. Sahota, P. P. 2005. Contaminants in Drinking Water. Research Report, Punjab Agricultaral University, Tribune Publications.

14. Cray, W. C. J., and H. W. Moon. 1995. Experimental infection of calves and adult cattle with Escherichia coli O157:H7. Appl. Environ. Microbiol. 61 (4) 1586-1590.

15. Singh, A., and G. A. Mefeters. 1992. Detection method for water borne pathogens. In R. Mitchell (ed.), Environmental microbiology. John Willey and sons Inc., New York

16. Nahar, A., M. M. Ahmed, and A. Chakraborty. (2011). A Quality Analysis of Dhaka WASA Drinking Water: Detection and biochemical characterization of the Isolates. J. Environ. Sci. Nat. Res. 4 (2): 41-49.

17. Munshi, S. K., M. M. Rahman, and R. Noor. 2012. Detection of virulence potential of diarrheagenic Escherichia coli isolated from surface water river surrounding Dhaka city. J. Bang. Acad. Sci. 36 (1): 109-122.

18. Noor, R., et al. 2013. Microbiological study of major sea fish available in local markets of Dhaka city, Bangladesh. J. Microbiol. Biotech. Food Sci. 2 (4): 2420-2430.

19. Johnson, T. and C. Case. 2010. Laboratory Experiments in Microbiology. Benjamin Cummings, San Francisco.

20. Bej, A. K., et al. 1990. Detection of coliform bacteria in water by polymerase chain reaction and gene probes. Appl. Environ. Microbiol. 56 (2): 307-314.

21. Tharannum, S., et al. 2009. Molecular confirmation of the presence of coliforms in drinking water using polymerase chain reaction. Kath. Uni. J. Sci. Eng. Tech. 5 (2): 130-136.

22. Goel, S., et al. 2007. Bacteriological quality of water samples of a tertiary care medical center campus in north western Himalayan region of India. Inter. J. Third World Med. 5 (1). DOI: 10.5580/10d5

23. Abera, S., et al. 2011. Bacteriological analysis of drinking water sources. Afr J. Microbiol. Res. 5 (18): 2638-2641.

24. Cappuccino, J. G., and N. Sherman. 1996. Microbiology- A laboratory manual. The Benjamin/Cummings Publishing Co., Inc., Menlo Park, California.

25. Alfrad, E., and B. Bensons. 2007. Microbiological Applications. McgrawHill Book Company., New York.

26. Bauer, A. W., W. M. M. Kirby, J. C. Sherris, and M. Tierch. 1966 Antibiotic susceptibility testing by a standardized single disc method. Ame. J. Clin. Patholog. 45 (4): 493-496.

27. Ferraro, M. J., W. A. Craig, and M. N. Dudley. 2001. Performance standards for antimicrobial susceptibility testing. NCCLS, Pennsylvania, USA 
28. Bennett, P. M. 2008. Plasmid encoded antibiotic resistance: Acquisition and transfer of antibiotic resistance genes in bacteria. Bri. J. Pharma. 153 (1): 347-357

29. Canton, R. 2009. Antibiotic resistance genes from the environment: A perspective through newly identified antibiotic resistance mechanisms in clinical setting. Euro. Soc. Clin. Microbiol. Infect. Dis. 15 (1): 20-25.

30. Hung, D. T., and B. B. Kaufman. 2010. The fast track to multidrug resistance. Mol. Cell Biol. 37 (3): 297-298.
31. Schwartz, T., W. Kohnen, B. Jansen, and U. Obst. 2003. Detection of antibioticresistant bacteria and their resistance genes in waste-water, surface water, and drinking water biofilms. FEMS Microbiol. Ecol. 43: 325-335.

32. Dutta, S., et al. 2013. Study of antimicrobial susceptibility of clinically significant microorganisms isolated from selected areas of Dhaka, Bangladesh. Bang. J. Med. Sci. 12 (1): 34-42.

33. Khan, S. I., F. Feroz, and R. Noor. 2013. Study of extended spectrum a-lactamase producing bacteria from urinary tract infection in Dhaka city, Bangladesh. Tzu Chi Medical Journal 25 (1): 39-42. 